\title{
A New Approach for Blood Pressure Monitoring based on ECG and PPG Signals by using Artificial Neural Networks
}

\author{
Younessi Heravi \\ Mohammad Amin \\ Department of Basic Sciences, \\ School of Medicine, North \\ Khorasan University of Medical \\ Sciences, Bojnurd, Iran
}

\author{
Maghooli Keivan \\ Department of Biomedical \\ Engineering, Science and \\ Research Branch, Islamic Azad \\ University,Tehran, Iran
}

\author{
Joharinia Sima \\ Department of Electrical \\ Engineering, Young \\ Researchers and Elite Club, \\ Bojnurd Branch, Islamic Azad \\ University, Bojnurd, Iran
}

\begin{abstract}
Background: Pulse transit time has been demonstrated as one of the potential parameters for a cuffless blood pressure measurement. The accuracy of this method depends on the initial calibration that is obtained by several measurements. The aim of this study was to employ artificial neural network in order to estimate the blood pressure based on PTT. PTT is defined as the time delay between the R-wave of the ECG and the peak of the pulse wave in the finger. To train the ANN for modeling the blood pressure, this study used a database containing 65 subjects. For each subject, BP was taken several times in different condition. The trained ANN was capable of establishing a function between the PTT and the BP as an input and a response, respectively. The results of estimating BP were compared with the results of sphygmomanometer method and the error rate was calculated. The absolute error and error percentage in systolic blood pressure between cuff method and the present method were $5.41 \pm 2.63 \mathrm{mmHg}$, $4.09 \pm 1.59 \%$ and for diastolic blood pressure were $7.01 \pm 2.52$ $\mathrm{mmHg}, 6.88 \pm 2.43 \%$. The results indicated that the BP measurement by cuff method and BP predicted with trained ANN differ by only less than $10 \%$. It is obvious that the neural network prediction fit properly to the cuff results. The results of proposed method were closely in agreement with the results of the sphygmomanometer cuff. So the present method could be applied as an effective tool for the blood pressure estimation.
\end{abstract}

\section{Keywords}

Blood Pressure monitoring, Pulse transit time, Artificial neural network.

\section{INTRODUCTION}

Blood pressure is measured either invasively by an intraarterial catheter or non-invasively by cuff sphygmomanometer [1]. Cuff method is less accurate than arterial catheter, but it is used in most case because of convenience for measuring blood pressure. Arterial blood pressure management is a major problem in modern medicine and health care [2]. Blood pressure measurement system is useful to a person who manages blood pressure. This system allows a people to measure blood pressure themselves everywhere by simple operation. The automatic blood pressure measurement systems based on cuff are pre-programmed to record usually at every 15-20 minutes, which can cause inconveniences at everyday activities and loss of important information between the measurements. Therefore, a continuous measurement of blood pressure is more desirable for a hypertensive to monitoring their blood pressure [3]. In recent years, a large amount of research activities were made for non-invasive continuous blood pressure monitoring. Compared to other methods, the pulse transit time (PTT) method shows excellent results in many studies [4-6]. PTT, or known as the inverse of pulse wave velocity, has been demonstrated as one of the potential parameters for a cuffless and non-invasive blood pressure measurement [7, 8]. PTT is the duration for a pressure pulse to propagate through a segment of the arterial tree. It is measured between the characteristic points of electrocardiogram and peripheral pressure pulse. Photoplethysmograph is one of the popular choices for peripheral pressure pulse. PTT is obtained by continues recording of ECG and pulse wave [9]. A number of authors obtained a non-linear relationship between PTT and blood pressure $[10,11]$, while a number of authors reported linear relations [12, 13]. For continuous measurement of blood pressure by this method, the constant coefficients are needed in addition to the continuous extraction of PTT [13]. The initial calibration is also required on each subject because of different physiological parameters, such as the blood density and the stiffness of the arterial wall [14-15]. Those parameters can be treated as constant for each personal subject and by doing several measurements the linear regression line is established and the coefficients are obtained. Then the blood pressure can be predicted based on the PTT. The prediction accuracy depends on the accuracy of the initial calibration. The number of measurements can be altered during the initial calibration. But several times of measurements were formal to be acceptable to obtain a reasonable regression line. So this method has some limitations of, which was the use of the cuff method for the reference blood pressure that could not be considered as the standard. Moreover this method depends on the cuff method for the initial calibration and initial calibration also depends on the several measurements in different conditions [16]. The aim of this study was to design a method which blood pressure is monitored continuously and without cuff. The system is also based on the PTT method as many other studies, but doesn't employ cuff for the initial calibration and use artificial neural network to get relation between PTT and blood pressure. 


\section{METHODS}

\subsection{PTT Extraction}

PTT is defined as the time delay between the R-wave of the ECG and the peak of the pulse wave in the periphery (finger). The R-wave was detected from a chest lead of the ECG using amplitude and slope criteria. The arrival of the pulse wave was defined by the peak value of the signal, which corresponds to the maximum part of the plethysmography signal [13].

\subsection{Data Collection}

In this study PTT was earned in normal condition and different physical activities. Moreover blood pressure was measured three times in 0 and \pm 90 degree of hand in all condition and proportional to it, PTT was earned. Physical activity included going up and down the stairs to increase the arterial blood pressure. The workload was kept until the sub maximal heart rate was achieved thereafter 5-7 minutes was considered for individuals to restore to normal condition. PTT, systolic and diastolic blood pressure was measured for each individual in the normal conditions, different physical activities and different degree of hand. All blood pressure measurements were taken by digital brachial blood pressure monometer (Citizen, BK202) on the left upper arm. Each subject was requested to perform several trials of measurements. Accordingly PTT and proportional to it, blood pressure was collected.

\subsection{Data Processing}

In the study, artificial neural network (ANN) model was developed to predict the blood pressure based on PTT. When the PTT was input to the ANN, the corresponding blood pressure value could be calculated. In order to validate the obtained results they were compared with the results obtained by cuff method. Because the sample data have very different orders of magnitude the input and output data was normalized before applying them to the neural network. This way the accuracy and processing speed of the neural network could be increased. In addition, more reliable predictions can be made. The input data (PTT) and output data (blood pressure) were normalized to range from 0 to 1 by Equation (1):

$$
D_{n}=\frac{D_{i}-D_{\min }}{D_{\text {max }}-D_{\text {min }}}
$$

Where $D_{n}$ and $D_{i}$ denote the normalized and real value, $D_{\text {min }}$ and $\mathrm{D}_{\max }$ denote the minimum and maximum real value in the database.

\subsection{Estimation Blood Pressure by ANN}

ANN is one of the artificial intelligence methods. ANNs attempt to find the desirable relation between the input and the corresponding output parameters during the learning process. Training is a process in which the network is trained by the presented samples. A neural network learns by determining the relation between the inputs and outputs. This process carried out by calculating the relative importance of them. The trained ANNs can predict the output of new unknown samples $[17,18]$. In this study, The PTTs were the inputs and the blood pressures were the corresponding outputs. These data were applied to train the networks in the learning process. The trained neural network acts as a function. This function predicts the blood pressure as output for any new PTT as an input. Therefore, changes in outputs which could be the result of different inputs can be estimated by the trained ANN. The model was implemented in MATLAB software. The ANN toolbox of MATLAB provides a comprehensive set of functions and a graphical user interface for the design, implementation, visualisation, and simulation of neural networks [19]. Network architecture, learning algorithms and activation function are the main components of an artificial neural network. These components affect the network performance. Therefore, for achieving the good performance of the network, these components must be determined carefully.

\subsection{ANN Design}

Network architecture is the number of network layers, number of neurons in each layer and connections between neurons. There is no general rule to select the optimum network architecture; so many architectures should be tried to pinpoint an appropriate one. For obtaining the desired results, the multilayer feed-forward back propagation was utilized. This network consists of three types of neuron layers that contain one input layer, one or several hidden layers and one output layer. Usually, for the training of this network the most popular learning algorithm called back propagation is applied. The back propagation algorithm method is based on learning the ANN by adjusting the connection weights in order to minimize the network errors of prediction (error between the desired and real output values). The network training is an iterative process. If the network error reduces to a minimum, the training process goes toward stability. Activation function determines the relationship between inputs and outputs of the nodes. There are different activation functions. The most popular ones such as LOGSIG and TANSIG were utilized to achieve the best performance. The ability of the neural network model to predict the blood pressure was evaluated by the mean square error (MSE) and coefficient of determination $\left(\mathrm{R}^{2}\right)$ between the measured and predicted data. The accuracy of neural network mode measured by the $\mathrm{R}^{2}$ parameter explains the model's goodness of fit $[20,21]$. The presented data was randomly divided into training, validating and testing the network with appropriate architecture and learning algorithms. Training data are presented to the network during training and adjusting the connection weights to minimize the network errors of prediction. Validation data are utilized to assess network generalization. Testing data have no effect on training and give an independent measure of network performance during and after the training. In this study, the different activation function effects were examined on the network performance. For obtaining the optimum architecture of network, the numbers of layers and neurons in each layer were increased step by step.

\section{RESULTS}

In this study 65 subjects participated ranging from 19 to 61 among them 37 were male and 28 were female. For each subject, blood pressure was taken several times (between 6 to 12 times) in different condition. The mean heart rate $77.236 \pm 9.872$ per min and the average height $1.624 \pm 0.068 \mathrm{~m}$. Data collection was started in June 2011 and finished in December 2012. Figure 1 shows the recording of ECG and PPG signals to calculate the PTT. 


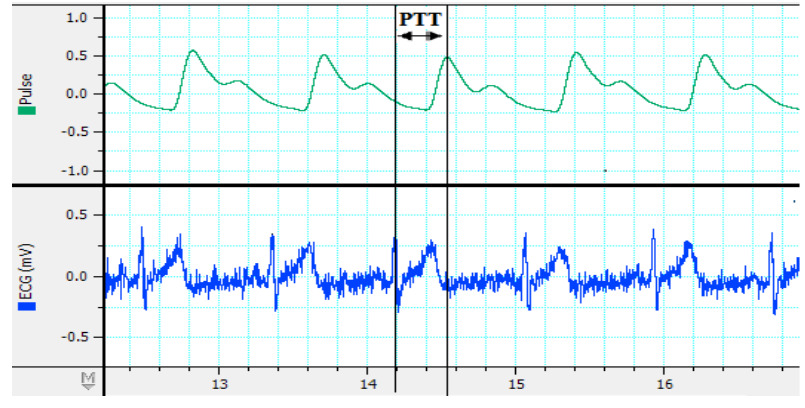

Fig 1: calculation of PTT by continuous recording of ECG and PPG signals.

The architecture of the optimal network model was feed forward with an input node, six nodes in the hidden layer and two output nodes. The number of output nodes in the output layer was 2 represented the systolic and diastolic blood pressure and the number of input nodes in the input layer is 1 represents the PTT. These inputs and corresponding outputs are applied as training patterns. Figure 2 illustrates the structure of the designed ANN. The total training time for the network was less than 15 seconds.

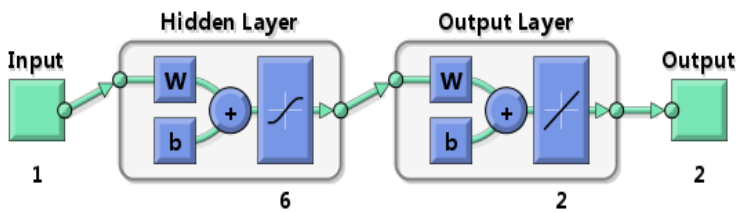

Fig 2: Used ANN structure

Figure 3 illustrates the circumstances of the learning process for optimum network model by the present sample using MSE on all epochs.

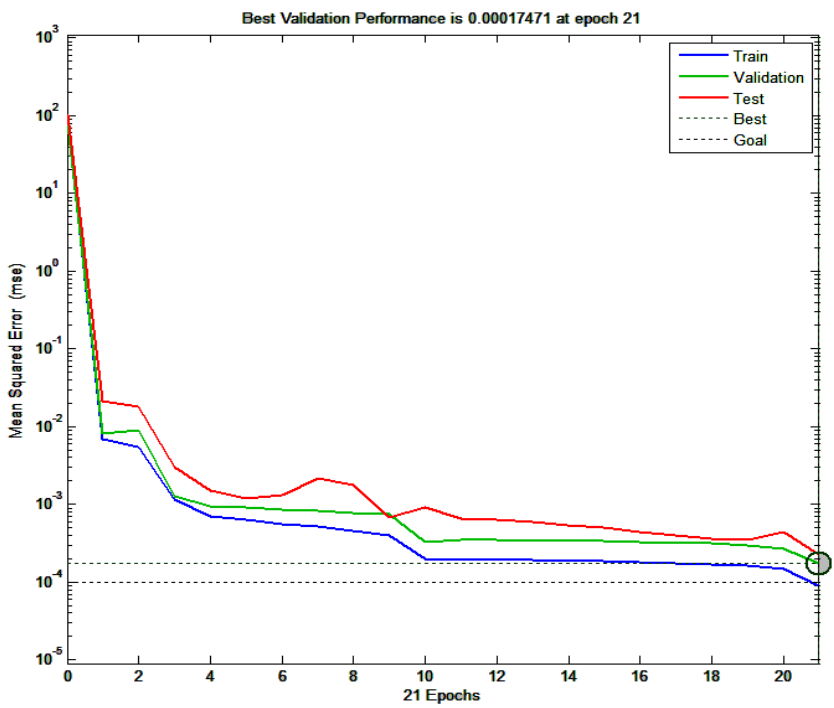

Fig3: The circumstances of optimum network learning process
This Figure shows that the training error has a small value on the last epoch. Furthermore, because of the low validation error the network has good generalisation ability, and because of the low testing error the network has an excellent performance during and after the training. The optimal network presents $\mathrm{MSE}=1.74 \mathrm{E}-4$ and $\mathrm{R} 2=0.86$ converging in 21 epochs with TANSIG and PURELIN activation function for hidden and output layers respectively. Figure 4 shows the relation and Pearson regression coefficient between target and prediction blood pressure.
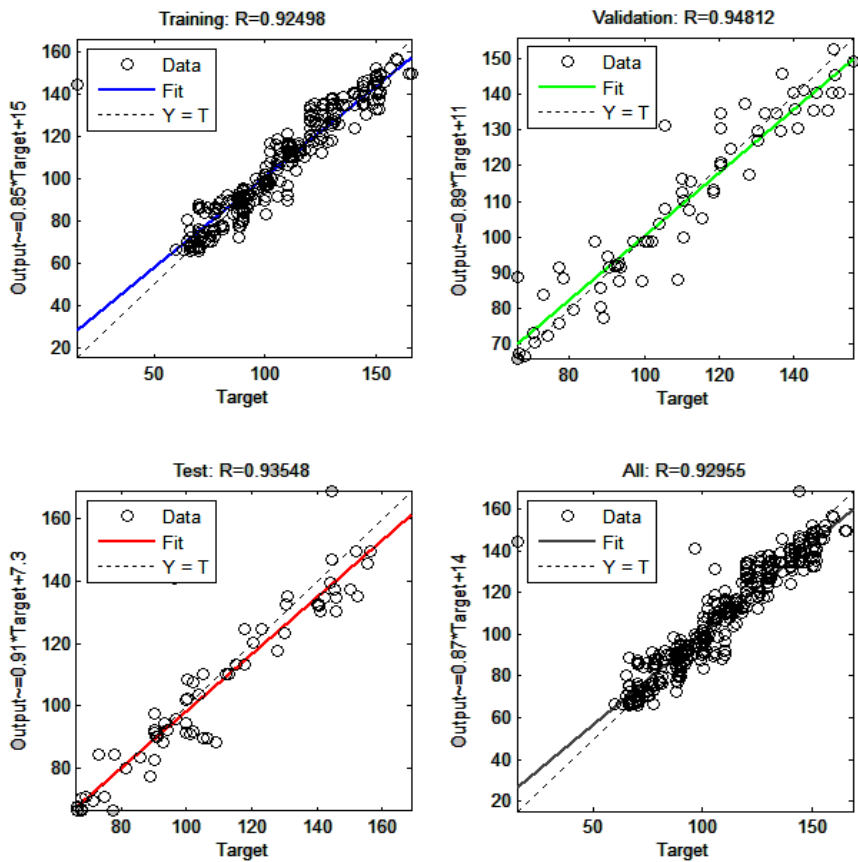

Fig 4: the relation and Pearson regression coefficient between target and prediction blood pressure 


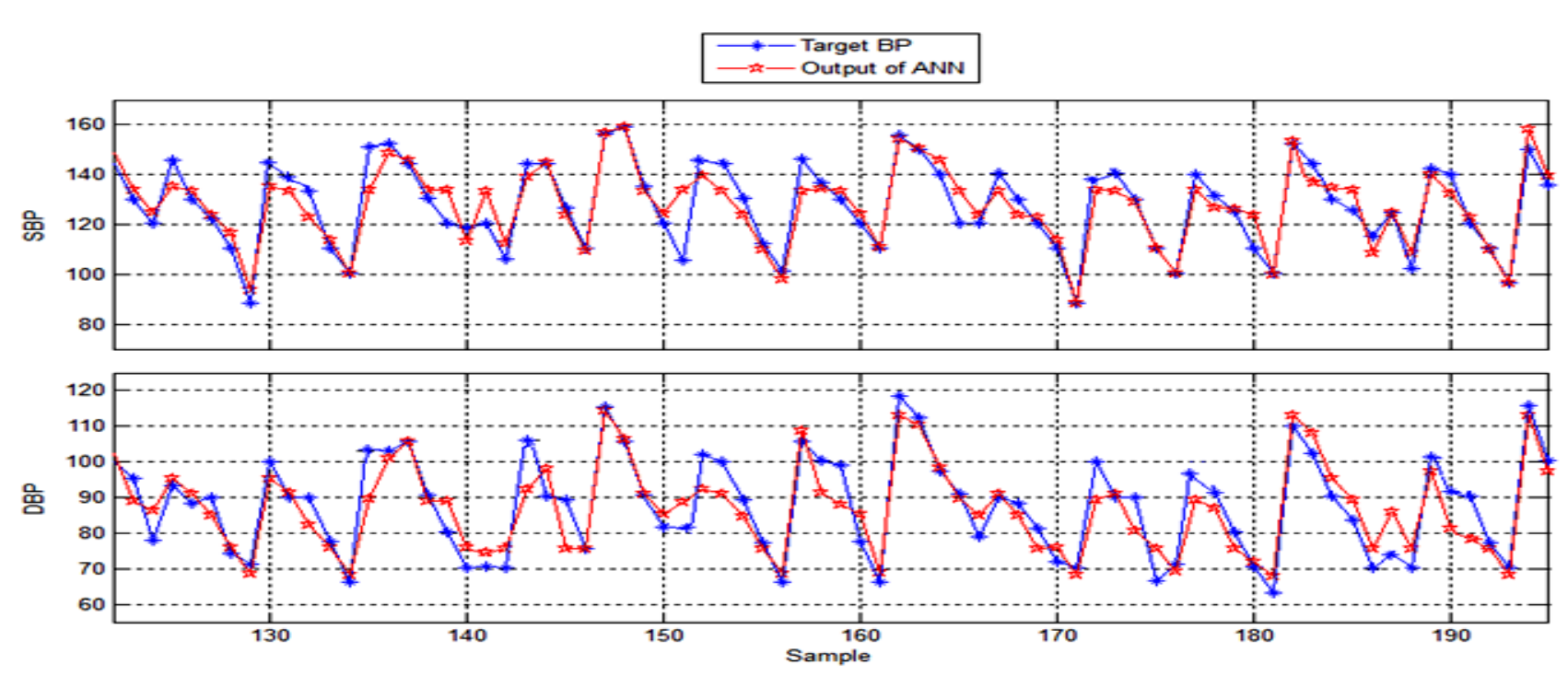

Fig 5: the prediction blood pressure (ANN output) and the blood pressure from cuff method (target blood pressure)

Figure 5 shows the blood pressure which obtained from the ANN and the blood pressure achieved by using sphygmomanometer cuff. Absolute error and error percentage between cuff method and trained ANN were 5.41 \pm 2.63 $\mathrm{mmHg}$ and $\% 4.09 \pm 1.59$ for systolic blood pressure and $7.01 \pm 2.52 \mathrm{mmHg}$ and $\% 6.88 \pm 2.43$ for diastolic blood pressure. By comparing these two sets of results, it has been found that the trained ANN could properly predict the blood pressure. The error in female subjects was $6.58 \pm 2.38$ and it was $6.25 \pm 2.26$ for male subjects. There was no significant relationship between male and female subject in the error. Also, in male and female subjects systolic blood pressure was measured more accurately than diastolic blood pressure.

\section{DISCUSSION}

This paper presents a new method for obtaining the cuffless and continuous blood pressure based on PTT method by trained ANN. Absolute error and error percentage between cuff method and trained ANN were $5.41 \pm 2.63 \mathrm{mmHg}$ and $\% 4.09 \pm 1.59$ for systolic blood pressure and $7.01 \pm 2.52 \mathrm{mmHg}$ and $\% 6.88 \pm 2.43$ for diastolic blood pressure. The results indicated that the blood pressure measurement by cuff method and blood pressure predicted with trained ANN differ by only less than $10 \%$. It is obvious that the neural network prediction could fit properly to the cuff results. The results showed that maximum error was $10.08 \mathrm{mmHg}$ for systolic blood pressure and 11.39 for diastolic blood pressure. The previous studies showed that systolic blood pressure could be measured accurately than diastolic blood pressure [13,22,23]. The results of trained ANN were in good arrangement with these studies. Also the error of systolic blood pressure in this study was obtained less than the previous study. In several studies blood pressure in male subjects measured with less error and higher correlation than female subjects [13,24]. In the blood pressure prediction by ANN was not significant relationship between male and female subjects. In other studies, by doing several measurements, the function was established between PTT and blood pressure $[1,5,16,25,26]$, the present study could predict blood pressure without several measurement and the results of it were better than other studies. For a model which has the smallest value of MSE and where R2 value is high (greater than 0.8), the model can be determined as the best [20], the present model had R2 $=0.86$ so it could fit property to blood pressure by PTT. Some studies obtained nonlinear relationship between PTT and blood pressure $[6,10,11]$ while in some other studies blood pressure was estimated based on PTT by The linear function [13,16,25]. The trained ANN can be a good model to calculate blood pressure based on PTT because the advantages of using ANNs are: the regression performs better when theory or experience indicates an underlying relationship, fast computation, ability to learn by means of the presentation samples, prediction of unknown samples; the flexibility affronts the noisy samples and performance will improve as sample size increases [27]. the estimating blood pressure accuracy in the study depends on the initial values of PTT and blood pressure, because the ANN was trained by this parameters so the blood pressure, PTT measurements should be performed with the standard devices to obtain highly accurate results. In this study, a model was considered for prediction of systolic and diastolic blood pressure. ANN was trained with PTT as an input and systolic and diastolic blood pressure as outputs while ANN could be trained with systolic and diastolic blood pressure separately and a model can be considered for each output. It can make the better results but increases model complexity and also processing time. Moreover increasing the trained dataset with PTT and blood pressure for more condition and especially in abnormal (hypertensive and hypotensive) subjects will be able to develop a good model to predict blood pressure. Also using Dynamic neural network and other pattern recognitions will be able to make better results.

\section{CONCLUSION}

A new approach is developed based on the ANN, in order to estimate the blood pressure based on ECG and PPG signals. The blood pressure which obtained from the proposed method is closely in agreement with the results achieved by using the sphygmomanometer cuff within less than $10 \%$ tolerance. In this study, the blood pressure calculations could be simply and rapidly done for any subject without several measurements in different condition for determining function. Only the process of training of the ANN needs some time (in this case less than $15 \mathrm{sec}$ ) after this process the trained ANN acts as a function and can predict the blood pressure for any new PTT. Consequently, the method presented herein could be applied as an effective tool for the blood pressure estimation. 


\section{ACKNOWLEDGMENT}

We would like to thank to all people in North Khorasan University of Medical Sciences, Bojnourd and Mashad Islamic Azad University that participated in the study and helped us to collect data.

\section{REFERENCES}

[1] Lass J, Meigas K, Karai D, Kattai R, Kaik J, Rossmann M. 2004 Continuous blood pressure monitoring during exercise using pulse wave transit time measurement, Proc. of the 26th Annual International Conference of the IEEE EMBS; 2239-2242.

[2] Campbell NR, Chockalingam A, Fodor JG, McKay DW. 1990 Accurate, reproducible measurement of blood pressure. CMAJ; 143:19-24

[3] Elena C, Guo C, Brenton A, Maxime C. 2013 Noninvasive continuous blood pressure monitoring: a review of current applications. Front. Med; 7(1): 91-101

[4] Maggi R, Viscardi V, Furukawa T, Brignole M. 2010 Non-invasive continuous blood pressure monitoring of tachycardic episodes during interventional electrophysiology. Europace ;12(11):1616-22.

[5] Chen W, Kobayashi T, Ichikawa S, Takeuchi Y, Togawa T. 2000 Continuous estimation of systolic blood pressure using the pulse arrival time and intermittent calibration. Med Biol Eng Comput; 38 (5): 569-74.

[6] Nitzan M, Khanokh B, Slovik Y. 2002 The difference in pulse transit time to the toe and finger measured by photoplethysmography. Physiol Meas: 23(1): 85-93.

[7] Chen Y, Wen C, Tao G, Bi M, Li G. 2009 Continuous and non-invasive blood pressure measurement: a novel modeling methodology of the relationship between blood pressure and pulse wave velocity. Ann Biomed Eng; 37(11):2222-33.

[8] Poon C Y, Zhang Y T, Liu Y B. 2006 Modeling of Pulse Transit Time under the Effects of Hydrostatic Pressure for Cuffless Blood Pressure Measurements, International Conference of IEEE in Medicine and Biology Society; 123-129.

[9] Pollak MH, Obrist PA. 1983 Aortic - radial pulse transit time and ECG Q- wave to radial pulse wave interval as indices of beat by beat blood pressure change. Apasychophysiology;20:21-8.

[10] Zheng D, Murray A. 2009 Non-invasive quantification of peripheral arterial volume distensibility and its non-linear relationship with arterial pressure. J Biomech; 42(8):1032-7.

[11] Callaghan FJ, Babbs CF, Bourland JD, Geddes LA. 1984 The relationship between arterial pulse-wave velocity and pulse frequency at different pressures. J Med Eng Technol; 8(1):15-8.

[12] Geddes LA, Voelz M, James S, Reiner D. 1981 Pulse arrival time as a method of obtaining systolic and diastolic blood pressure indirectly. Med Biol Eng Comput;19(5):671-2.

[13] Younessi Heravi MA, Khalilzadeh MA, Joharinia S. 2014 Continous and cuffless blood pressure monitoring using ECG and $\mathrm{SpO} 2$ signals. J Biomed Phys Eng; $4(1): 27-32$.

[14] Shin SH, Park YB, Rhim HW, Yoo YS, Park YJ, Park DH. 2005 Multibody dynamics in arterial system," Journal of Mechanical Science and Technology; 19(1):343-349.

[15] James D, Lisa Greenstadt L, Shapiro D. 1983 Pulse Transit Time and Blood Pressure: An Intensive Analysis, Psychophysiology; 20(1): 45-49.

[16] Younessi Heravi MA, Khalilzadeh MA. 2014 Designing and Constructing an Optical System to measure Continuous and Cuffless Blood Pressure Using Two Pulse Signals. Iranian Journal of Medical Physics; 10(4): 204-212

[17] Christopher Bishop M. 1995 Neural networks for pattern recognition. Oxford University, USA.

[18] Haykin S. 1999 Neural networks, a comprehensive foundation. Prentice Hall International Editions.

[19] Demuth H, Beale M, Hagan M. 2007 Neural network toolbox 5 user's guide. The Mathworks, Natick.

[20] Kozma R, Sakuma M, Yokoyama Y, Kitamura M. 1996 On the accuracy of mapping back propagation with forgetting. Neurocomp; 13(2):295-311.

[21] Kasabov NK. 1998 Foundations of neural networks fuzzy systems and knowledge engineering. MIT, Cambridge.

[22] Poon CCY, Zhang YT. 2005 Cuff- less and non invasive measurements of arterial blood pressure by pulse transit time. Proc. of the 27th Annual International Conference of the IEEE EMBS.

[23] Payne RA, Symeonides CN, Webb DJ, Maxwell SR. 2006 Pulse transit time measured from the ECG: an unreliable marker of beat-to-beat blood pressure. J Appl Physiol; 100(1):136-41.

[24] Allen J, A Murray A. 2002 Age-related changes in peripheral pulse timing characteristics at the ears, fingers and toes, Journal of Human Hypertension; 16: 711-717.

[25] Zong W, Moody GB, Mark RG. 1998 Effects of vasoactive drugs on the relationship between ECG pulse wave delay time and arterial blood pressure in ICU patients. Comput Cardiol; 25:673-6.

[26] Youngzoon Y, Jung HC, Gilwon Y. 2009 Nonconstrained Blood Pressure Monitoring Using ECG and PPG for Personal Healthcare. J Med Syst; 33:261-266.

[27] Soltane M, Ismail M, Abdul Rashid ZA. 2004 Artificial neural networks (ANN) approach to PPG signal classification. International journal of computing \& information sciences ; 2(2): 58-65. 